\title{
Recent developments of smart electromagnetic absorbers based polymer-composites at gigahertz frequencies
}

\begin{abstract}
The rapid increase in electromagnetic interference has received a serious attention from researchers who responded by producing a variety of radar absorbing materials especially at high gigahertz frequencies. Ongoing investigation is being carried out in order to find the best absorbing materials which can fulfill the requirements for smart absorbing materials which are lightweight, broad bandwidth absorption, stronger absorption etc. Thus, to improve the absorbing capability, several important parameters need to be taken into consideration such as filler type, loading level, type of polymer matrix, physical thickness, grain sizes, layers and bandwidth. Therefore, this article introduces the electromagnetic wave absorption mechanisms and then reveals and reviews those parameters that enhance the absorption performance.
\end{abstract}

Keyword: Radar absorbing materials; High gigahertz frequencies; Absorption mechanism; Reviews 